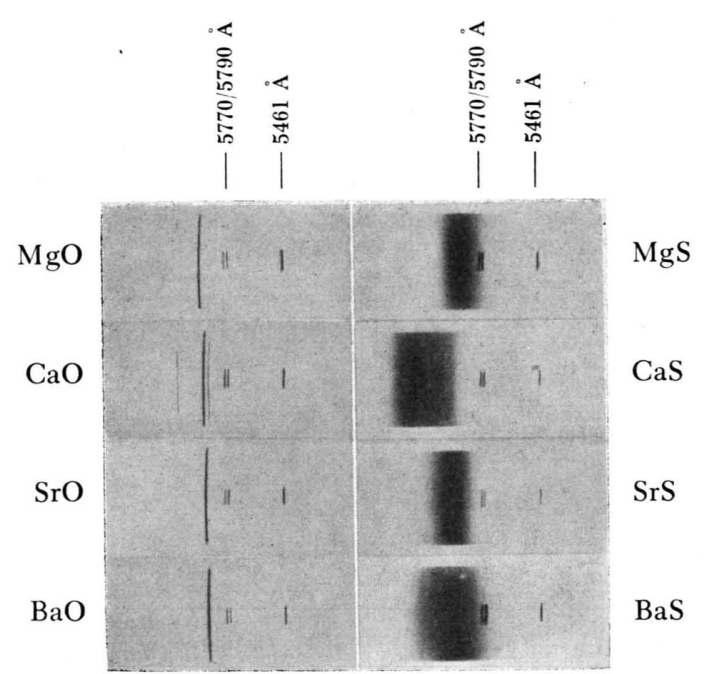

Abb. 1. Emissionsspektren des $\mathrm{Eu}$ in Erdalkali-Oxyden und -Sulfiden.

Bemerkenswert ist, daß im Spektrum des $\mathrm{Eu}^{+++}$eine einzige Linie die anderen an Intensität bedeutend übertrifft. D e u t s c h b e i n ${ }^{5}$ zeigte, daß der dieser Linie entsprechende Übergang mit $\Delta I=1$ in elektrischen Feldern der Symmetrie, wie sie am Ort eines Kations im $\mathrm{NaCl}$ Gitter anzutreffen ist, allein encliche Übergangswahrscheinlichkeit haben sollte. Das Überwiegen dieser Linie weist darauf hin, daß die der Rechnung wesentlich zugrunde liegende Annahme ungestörter Zentralsymmetrie bezüglich des Störions einigermaßen gerechtfertigt erscheint.

5 O. Deutschbein, in ,Leuchten und Struktur fester Stoffe“, herausgeg. v. R. To m a s c h e k, Oldenbourg, München 1942, S. 179.

\section{Über Störstellen, die in Erdalkalichalkogeniden durch Einbau Seltener Erden hervorgerufen werden}

\author{
Von Peter Brauer \\ Osram-Studiengesellschaft Mosbach (Baden)
}

(Z. Naturforschg. 6 a, 562-563 [1951]; eingeg. am 4. Sept. 1951)

Baut man Seltene Erden (SE) in sehr geringer Konzentration in Erdalkalichalkogenide ein, so nehmen sie im allgemeinen den Status eines dreiwertigen Ions im Wirtsgitter an, denn die Spektren der entstehenden Phosphore sind im wesentlichen die der reinen Salze der dreiwertigen $\mathrm{SE}$, wie $\mathrm{T}$ o m a s che k und $\mathrm{Deuts} \mathrm{chbein}{ }^{1}$ gezeigt haben. Eine Ausnahme bildet das verhältnismäßig leicht reduzierbare $\mathrm{Eu}$, das in $\mathrm{MgO}^{2}$ und $\mathrm{CaO}^{3}$ zwar das charakteristische Linienspektrum des $\mathrm{Eu}+++$ zeigt, in $\mathrm{CaS}$, $\mathrm{SrS}^{4}$ und $\mathrm{SrSe}^{5}$ dagegen im langwelligen Teil cles sichtbaren Gebiets eine breite diffuse Bande, die wir dem $\mathrm{Eu}^{++}$zugeschrieben haben ${ }^{4}$, worin $\mathrm{W}$ a $\mathrm{rd}^{6}$ mit uns übereinstimmt. Das gleichfalls - aber schwerer - reduzierbare $\mathrm{Sm}$ ist in allen Erdalkalioxyden und -sulfiden dreiwertig 7 .

Dieses Verhalten läßt sich nun verstehen, wenn man gittertheoretisch die Energietönung einer Reaktion berechnet, bei der die dreiwertige $\mathrm{SE}$ in clie zweiwertige übergeführt würde. Bei der Auswahl der der Rechnung zugrunde zu legenden chemischen Gleichung bedarf es vor allem einer Annahme über die ladungskompensieren-

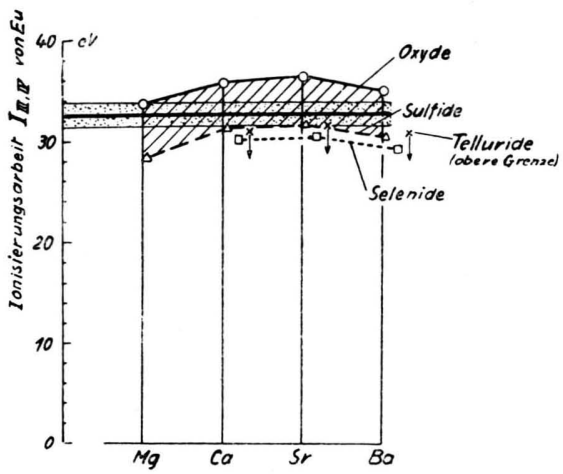

Abb. 1. Theoretische Kurven der Ionisierungsarbeit IIII, IV von Eu. Unterhalb einer Kurve ist in dem betreffenden Wirtsgitter $\mathrm{Eu}+++$, oberhalb $\mathrm{Eu}++$ stabil.

den ergänzenden Störstellen im Falle der $\mathrm{SE}^{+++}+\mathrm{Da}$ $\mathrm{B}$ a n ks und $\mathrm{W}$ a $\mathrm{rd}^{8}$ für $\mathrm{Ce}^{++}+$in Erdalkalisulfiden zeigten, daß sich Schottkysche Kationenlöcher bilden, nahmen wir dies vorläufig als für Eu und $\mathrm{Sm}$ geltend an und formulierten demgemäß versuchsweise [s. unten (1)].

Bei der gittertheoretischen Berechnung der Ausbauarbeiten der Wirtsionen und der Störionen aus dem Kristall wurden Korrekturen der einfachen halbklassischen Gittertheorie nach früheren Erfahrungen ${ }^{9}$ berücksichtigt. Die Gitterverzerrung und -polarisation infolge Ladungs- und Größendifferenz zwischen Stör- und Wirtsgitter-Ionen war von entscheidender Bedeutung für das Ergebnis. Die Berechnung der Energietönung $c$, deren Vorzeichen etwas über die Richtung der durch (1) formulierten Reaktion und damit über die zu erwartende Wertigkeit der SE aussagen würde, ist darum vorerst nicht vollständig möglich, weil die Ionisierungsarbeit des $\mathrm{Eu}++$ und $\mathrm{Sm}^{++}$

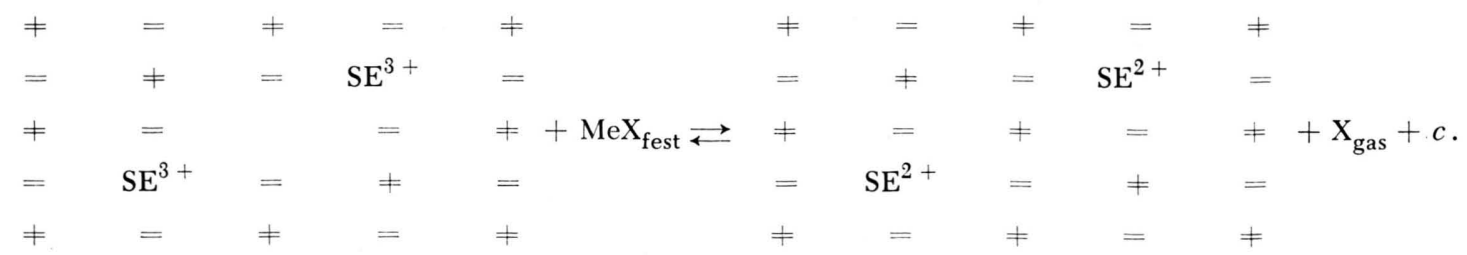

Hierin bedeuten Me ein Erdalkalimetall, $\mathrm{X}$ ein Chalkogen, $\neq$ und $=$ deren zweiwertige Ionen und $c$ die Energietönung. 
unbekannt ist. Wir berechneten statt dessen diese für die Gleichung (1), d.h. wir setzten in (1) $c=0$ und $=$ statt $\rightleftarrows$, und zwar für Eu bzw. Sm in den im NaCl-Gitter kristallisierenden Erdalkalichalkogeniden. Die weiteren gittertheoretischen Voraussetzungen und die Methoden waren denen analog, die bei der Berechnung der Energie der $\mathrm{Tl}+$-Störionen in Alkalihalogeniden $\mathbf{1 0}$ benutzt wurden; Einzelheiten folgen demnächst.

Die so berechneten hypothetischen Ionisierungsarbeiten sind in Abb. 1 aufgetragen. Deren Sinn ist also z. B. folgender: Liegt die SE mit ihrer wirklichen Ionisierungsarbeit oberhalb der „Oxydkurve“, so ist der zweiwertige Zustand im Oxyd stabil; liegt sie darunter, so ist es der dreiwertige; entsprechendes gilt für die "Sulfidkurve“ usw. Man erkennt: die Oxyd- und die Sulfidkurve liegen so, daß $\mathrm{Eu}^{++}$nur ein geeignetes Ionisierungspotential (nach der vorläufig nur groben Näherung $\sim 32 \mathrm{eV}$ ) zu haben braucht, um in allen Oxyden dreiwertig, in allen Sulfiden, Seleniden und Telluriden dagegen zweiwertig zu sein. Sm, von dem bekannt ist, daß es in allen Oxyden und Sulfiden dreiwertig ist, müßte ein $I_{\text {III/IV }}<28 \mathrm{eV}$ haben. Der Absolutwert der $I$ ist zwar nicht unvernünftig; doch muß damit gerechnet werden, daß er sich bei Wiederholung der Rechnung mit höherer Näherung noch etwas verschiebt.

Außerdem ergeben sich Voraussagen für die noch nicht untersuchten Erdalkalichalkogenid-SE-Phosphore. Danach müßte $\mathrm{Eu}^{+++}$in $\mathrm{SrO}$ und $\mathrm{BaO}$ erwartet werden, dagegen $\mathrm{Eu}^{+}+$in $\mathrm{MgS}, \mathrm{BaS}$, den Erdalkali-Seleniden und -Telluriden.

1 R. Tom a s chek u. O. D e u t s c h bein, Physik. Z. 34, 374 [1933].

2 O. Deutschbein, in "Leuchten und Struktur fester Stoffe“, herausgeg. von R. T o m a s c h e k, Oldenbourg, München 1942, S. 179.

3 G. U r b a in, Ann. Chimie (8) 18, 317 [1909].

$4 \mathrm{P}$. B r a u e r, Über sens. Phosphore III, Zentr. wiss. Berichtswes. 1943, Nr. 1695/3; Z. Naturforschg. 1, 70 [1946].

5 R. W a r d, in "Solid Lumin. Materials“, CornellSymp. 1946, New York 1948, S. 22.

6 R. W a r d, Diskussionsbemerk. daselbst 5, S. 299.

7 R. T o m a s chek, Physik. Z. 33, 878 [1932].

8 E. B a n k s u. R. W a rd, J. electrochem. Soc. 96, 297 [1949].

9 P. B r a u e r, Z. Naturforschg. 6a, 255 [1951].

10 P. B r a u e r, Z. Naturforschg. 6a, 560 [1951].

\section{Ein ungewöhnlicher Anstieg der Ultrastrahlung}

Von A. Sittkus und Dieter Ganz

Physikalisches Institut der Universität Freiburg i. Br.

(Z. Naturforschg. 6 a, 563-564 [1951]; eingeg. am 4. Sept. 1951)

In Freiburg $\left(48^{\circ} \mathrm{N}, 8^{\circ} \mathrm{E}\right)$ werden Dauerregistrierungen der Ultrastrahlung (U.S.) in einer Bergstation (Schauinsland $1200 \mathrm{~m})$ und einer Talstation $(300 \mathrm{~m})$ durchgeführt. Die Höhenstation mißt die Ionisation in einer $60-l$-Druckkammer hinter allseitig $5 \mathrm{~cm}$ Blei. In der Talstation wird eine 500-l-Druckkammer hinter allseitig $100 \mathrm{~g} / \mathrm{cm}^{2}(10 \mathrm{~cm}$ Eisen $+10 \mathrm{~cm}$ Barytbeton) verwendet. Der Energieverlust der U.S.-Teilchen zwischen den beiden Meßorten beträgt

\begin{tabular}{|c|c|c|}
\hline \multirow[t]{2}{*}{ MGZ } & \multicolumn{2}{|c|}{$\begin{array}{c}\text { Abweichung } \\
\text { vom Bezugswert in } \%\end{array}$} \\
\hline & Schauinsland & Freiburg \\
\hline $10^{00}-10^{15}$ & $+21,0$ & $\begin{array}{l}+8,8 \\
\end{array}$ \\
\hline $10^{15}-10^{30}$ & 29,7 & 12,1 \\
\hline $10^{30}-10^{45}$ & 24,5 & 15,1 \\
\hline $10^{4.5}-11^{00}$ & 18,9 & 4,3 \\
\hline \multirow[t]{4}{*}{$11^{00}$} & 17,5 & 11,5 \\
\hline & 16,6 & 14,4 \\
\hline & 13,8 & 12,1 \\
\hline & 21,0 & 14,8 \\
\hline \multirow[t]{4}{*}{$12^{00}$} & 22,3 & 16,5 \\
\hline & 31,9 & 5,1 \\
\hline & 28,4 & 12,2 \\
\hline & 27,4 & 7,9 \\
\hline \multirow[t]{3}{*}{$13^{00}$} & 62,7 & 11,8 \\
\hline & 55,4 & 0,8 \\
\hline & $\begin{array}{l}82,7 \\
67,8\end{array}$ & $\begin{array}{r}9,1 \\
15,0\end{array}$ \\
\hline \multirow[t]{4}{*}{$14^{00}$} & 76,9 & 11,2 \\
\hline & 53,9 & 6,6 \\
\hline & 59,5 & 12,9 \\
\hline & 56,5 & 8,9 \\
\hline \multirow[t]{4}{*}{$15^{00}$} & 51,9 & 8,2 \\
\hline & 38,0 & 11,0 \\
\hline & 37,5 & 15,0 \\
\hline & 19,7 & 15,6 \\
\hline \multirow[t]{4}{*}{$16^{00}$} & 29,8 & 6,4 \\
\hline & 10,4 & 11,0 \\
\hline & 29,8 & 8,9 \\
\hline & 18,7 & 9,6 \\
\hline $17^{00}-17^{15}$ & 26,3 & 18,9 \\
\hline $17^{15}-17^{30}$ & 13,9 & 10,3 \\
\hline $17^{30}-17^{45}$ & 30,9 & 7,0 \\
\hline $17^{45}-18^{00}$ & $+17,6$ & $+11,2$ \\
\hline stat. Fehler & $\sim 6^{\circ} \% 0$ & $\sim 3 \%$ \\
\hline
\end{tabular}

Tab. 1. Ultrastrahlung in $\%$.

bei Berücksichtigung der Luftdruckdifferenz (100 mb) und der Panzerunterschiede $\left(45 \mathrm{~g} / \mathrm{cm}^{2}\right)$ unter Annahme normaler Ionisationsverluste $1,8 \cdot 10^{3} \mathrm{eV}$. Tab. 1 gibt den Verlauf der auf gleichen Luftdruck reduzierten Viertelstundenwerte der Intensität auf dem Schauinsland (Spalte 2) und in Freiburg (Spalte 3) wieder. Um $13 \mathrm{~h} \mathrm{MGZ} \mathrm{beginnt} \mathrm{in} \mathrm{der}$ Bergstation eine starke Zunahme der Intensität, wobei um etwa $14 \mathrm{~h}$ U.S.-Werte von $6 \%$ über Normal erreicht werden. Danach sinkt die Intensität bis etwa $16 \mathrm{~h}$ monoton auf normale Werte ab. In der Talstation ist von dem Effekt nichts zu beobachten, die Registrierung zeigt gainz normale Strahlungsschwankungen an. Der gemessene Effekt läßt sich unter der Annahme erklären, daß die Sonne in der fraglichen Zeit eruptiv U.S. so niederer Energie ausgesandt hat, daß die Strahlung in der Höhe noch beobachtet werden konnte, daß die Energie aber nicht mehr ausreichte, um in großer Tiefe wirksam zu werden 1. Eine rohe Berechnung der Energie der Eruptionsteilchen am Atmosphärenscheitel ergibt für senkrechten Einfall und Energieabbau allein durch Ionisationsverluste etwa $1,3 \cdot 10^{9} \mathrm{eV}$. Dieser Wert ist wegen Nicht- 